\title{
AUTOCUIDADO EM DIABETES POR USUÁRIOS DE UM SER- VIÇO DE ATENÇÃO PRIMÁRIA
}

\author{
SELF CARE ON DIABETES OF USERS IN A PRIMARY HEALTH CARE SYSTEM \\ AUTOCUIDADO DE LA DIABETES POR USUARIOS DE UNSERVICIO DE ATENCIÓN PRIMARIA
}

Mery Anne Epifânio Pereira ${ }^{1}$, Mira Wengert ${ }^{2}$, Letícia Gabriella Pereira Carvalho de Sousa ${ }^{3}$, Fernanda Alencar de Souza ${ }^{4}$, Mateus Silva Carvalho ${ }^{5}$, Lilian Dias Bernardo ${ }^{6}$

\begin{abstract}
RESUMO
O objetivo do estudo foi identificar o engajamento de pessoas com diabetes mellitus em atividades de autocuidado. Foi realizado um estudo descritivo transversal, com 42 pessoas com diabetes que frequentavam o projeto de educação e promoção em saúde aos diabéticos e hipertensos em uma clínica da família no Rio de Janeiro. Os dados revelaram um bom nível de autocuidado nos pacientes, especialmente nas atividades de gerenciamento medicamentoso e cuidado com os pés. No entanto, o domínio que corresponde à atividade física e o item de avaliar o açúcar no sangue receberam menores adesões. Tais resultados servem de subsídios para a organização, planejamento e execução de ações interprofissionais voltadas à promoção e educação em saúde dos diabéticos.
\end{abstract}

Palavras-Chave: Autocuidado; Diabetes mellitus; Autogestão.

\section{ABSTRACT}

The aim was to identify the engagement of people with diabetes mellitus in self-care activities. A cross-section al descriptive study was carried out with 42 people with diabetes who attended the health education and promotion project for diabetic sand hypertensive patients at a family clinic in Rio de Janeiro. The data revealed a good level of self-care in patients, especially in medication management and foot care activities. However, the domain that corresponded to physical activity and the item to measure blood glucose received less ad herence. Such results serve as subsidies for the organization, planning and execution of interprofessional actions aimed at the promotion and health education of diabetics.

Keywords: Self-care; Diabetes mellitus; Self-management.

\section{RESUMEN}

El objetivo del estúdio fue identificar la participación de las personas con diabetes mellitus em las actividades de autocuidado. Se realizóun estúdio descriptivo transversal con 42 personas con diabetes que asistieron al proyecto de educación y promoción de lasalud para diabéticos e hipertensos en una clínica familiar em Río de Janeiro. Los datosrevelaronunbuennivel de autocuidado enlos pacientes, especialmente enel manejo de medicamentos y actividades de cuidado de los pies. Sin embargo, eldominio que corresponde ala actividad física y elítem para evaluare la zúcaren sangre recibieron menos adherencia. Dichos resultados sirven como subsidios para laorganización, planificación y ejecución de acciones interprofesionales dirigidas a lapromoción y educación para lasalud de los diabéticos.

Palabras Clave: Autocuidado; Diabetes mellitus; Automanejo.

\footnotetext{
${ }^{1}$ Instituto Federal do Rio de Janeiro, Rio de Janeiro, Brasil. (0000-0003-4502-2986)

${ }^{2}$ Instituto Federal do Rio de Janeiro, Rio de Janeiro, Brasil. (0000-0002-6803-6662)

${ }^{3}$ Instituto Federal do Rio de Janeiro, Rio de Janeiro, Brasil. (0000-0002-4792-4791)

${ }^{4}$ Instituto Federal do Rio de Janeiro, Rio de Janeiro, Brasil. (0000-0002-4328-8572)

${ }^{5}$ Instituto Federal do Rio de Janeiro, Rio de Janeiro, Brasil. (0000-0002-2545-5808)

${ }^{6}$ Instituto Federal do Rio de Janeiro, Rio de Janeiro, Brasil. (0000-0001-5234-4225)
} 


\section{INTRODUÇÃO}

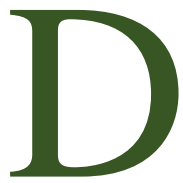

iabetes mellitus (DM) é um relevante problema de saúde pública e atinge, atualmente no Brasil, um quantitativo aproximado de 16 milhões de pessoas entre 20 e 79 anos, com estudos prospectivos para aumento dessa condição de saúde. Adicionalmente, o diabetes tem um relevante impacto econômico nos países e nos sistemas de saúde, decorrente de uma maior utilização desses serviços, perda de produtividade e cuidados prolongados requeridos para tratar esta doença crônica e suas possíveis comorbida$\operatorname{des}^{1}$.

Dentre as complicações mais prevalentes estão as doenças cardiovasculares, a neuropatia, nefropatia e retinopatia, com potenciais para a redução da funcionalidade, da qualidade de vida e elevação da taxa de mortalidade. No entanto, a aderência do paciente ao tratamento é fator essencial para o controle do diabetes e para a prevenção de complicações ${ }^{2}$.

No tratamento, diversas estratégias de promoção da saúde devem ser incorporadas no cotidiano das pessoas com diabetes, em que se destaca um programa de educação continuada voltado para o autocuidado ${ }^{2}$. Ações interprofissionais devem ser incluídas, contemplando o gerenciamento medicamentoso, a adoção de uma alimentação saudável, prática regular de atividade física, cuidados com a pele (sobretudo com os pés), moderação no uso de álcool e abandono do tabagismo, a fim de promover modificações para um estilo de vida mais saudável e maior controle desta condição de saúde ${ }^{3,4}$.

O sucesso do tratamento depende de uma equipe profissional que envolva ativamente a pessoa diabética (e sua família) na construção do plano de cuidados, mostrando ao indivíduo que ele é corresponsável pelo seu tratamento e que o conhecimento da condição de saúde reflete no comprometimento com o gerenciamento de sua saúde ${ }^{4}$. Nesse contexto, esta pesquisa buscou identificar o engajamento de pessoas com diabetes mellitus em atividades de autocuidado.

\section{METODOLOGIA}

Foi realizado um estudo descritivo transversal com abordagem quantitativa. Participaram 42 pessoas diabéticas ou pré-diabéticas que frequentavam o projeto de educação e promoção em saúde aos diabéticos e hipertensos, em uma clínica da família da zona oeste do Rio de Janeiro. Foram excluídos da pesquisa os pacientes somente hipertensos.

Para a coleta de dados, foram aplicados o questionário sociodemográfico para caracterizar a amostra e o Questionário de Atividades de Autocuidado com o Diabetes (QAD) para identificar a adesão ao tratamento por meio do nível de engajamento em atividades de autocuidado. Este questionário é a versão traduzida, adaptada e validada para o contexto brasileiro do Summary of Diabetes SelfCare Activities Questionnaire ${ }^{5}$.

O QAD é um instrumento padronizado composto por 18 itens que avaliam sete dimensões: alimentação geral, alimentação específica, atividade física, monitorização da glicemia, cuidados com os pés, gerenciamento medicamentoso e hábitos tabágicos. Ao responderem, os participantes destacam a frequência em que cada item foi realizado na última semana, com respostas variando de zero a sete dias, em que a maior frequência está associada a hábitos que podem controlar o diabetes e modificar o curso da doença. Somente duas questões - ingestão de alimentos ricos em gordura e ingestão de doces - precisam ter seus resultados ponderados, pois quanto menor a frequência, melhor o autocuidado e a adesão ao tratamento ${ }^{8}$. Durante a aplicação do questionário, os pesquisadores informavam ao participante que, se nos últimos sete dias ele estivesse se sentindo adoecido, que era para se pensar nas respostas considerando a semana mais recente sem a presença de adoecimento ${ }^{5}$.

A coleta de dados foi feita em dois formatos: presencialmente na clínica da família antes da pandemia do novo coronavírus (SARS-CoV-2) e por meio de contato telefônico com usuários que estavam afastados do serviço de atenção básica, devido às medidas restritivas de distanciamento físico 
impostas pela pandemia. Para análise dos dados, foi utilizada a estatística descritiva, incluindo índices de tendência central (média) e de dispersão (desvio e erro padrão), bem como frequência.

No que tange às considerações éticas, a pesquisa foi aprovada pelo Comitê de Ética em Pesquisa, assim como pela Secretaria Municipal de Saúde do Rio de Janeiro - SMS/RJ, sob pareceres do CEP-IFRJ $\mathrm{n}^{\mathrm{o}} 3.785 .722$ e CEP-SMS/RJ $\mathrm{n}^{\mathrm{o}}$ 3.908.547. Os participantes assinaram um termo de consentimento livre e esclarecido para conscientização dos objetivos da pesquisa. Para assegurar o anonimato e a confidencialidade das informações, os indivíduos receberam um número de codificação.

\section{RESULTADOS}

Para caracterizar a amostra, foi construída a tabela 1, que apresenta a distribuição das variáveis sociodemográficas dos 42 participantes da pesquisa.

Tabela 1 - Variáveis sociodemográficas dos participantes com Diabetes Mellitus

\begin{tabular}{|c|c|c|c|}
\hline Variáveis & Categoria & $\mathbf{N}$ & $\%$ \\
\hline \multicolumn{4}{|l|}{ Sexo } \\
\hline & Masculino & 8 & 19,05 \\
\hline & Feminino & 34 & 80,95 \\
\hline \multicolumn{4}{|l|}{ Idade } \\
\hline & Idoso & 22 & 52,38 \\
\hline & Adulto & 20 & 47,62 \\
\hline \multicolumn{4}{|l|}{ Estado Civil } \\
\hline & Solteiro & 11 & 26,19 \\
\hline & Casado & 17 & 40,48 \\
\hline & Viúvo & 10 & 23,81 \\
\hline & Separado & 4 & 9,52 \\
\hline \multicolumn{4}{|l|}{ Escolaridade } \\
\hline & Sem escolaridade & 5 & 11,90 \\
\hline & $\begin{array}{l}\text { Ensino fundamental } \\
\text { incompleto }\end{array}$ & 17 & 40,48 \\
\hline & $\begin{array}{l}\text { Ensino fundamental } \\
\text { completo }\end{array}$ & 8 & 19,05 \\
\hline & $\begin{array}{l}\text { Ensino médio com- } \\
\text { pleto }\end{array}$ & 8 & 19,05 \\
\hline & $\begin{array}{l}\text { Graduação incom- } \\
\text { pleta }\end{array}$ & 2 & 4,76 \\
\hline & Graduação completa & 2 & 4,76 \\
\hline
\end{tabular}

Ocupação

\begin{tabular}{cccc} 
Aposentado & 20 & 47,62 \\
Pensionista & 5 & 11,90 \\
Trabalhador & 8 & 19,05 \\
& Do Lar & 8 & 19,05 \\
& Desempregado & 1 & 2,38 \\
\hline Total & & 42 & 100
\end{tabular}

Fonte - Elaborada pelos autores

Os entrevistados, em sua maioria, eram do sexo feminino (80,95\%), casadas e aposentadas, seguidas de trabalhadoras ou donas de casa. No que tange à escolaridade, a maior parte dos participantes possuíam ensino fundamental incompleto $(40,48 \%)$, seguidos de fundamental completo e médio completo $(19,05 \%)$. Das comorbidades apresentadas, os participantes declararam possuir em sua maioria a hipertensão arterial sistêmica e doenças reumáticas. Dentro da amostra, seis pessoas eram pré-diabéticas, uma era diabética do tipo $1 \mathrm{e}$ 35 eram diabéticas tipo 2. Dentre elas, 15 eram insulínicas.

$\mathrm{Na}$ avaliação da aderência dos participantes às atividades de autocuidado investigadas pelo QAD, as frequências com suas médias de adesão por dias da semana e seus respectivos desvios e erros padrão são apresentados na tabela 2 .

Tabela 2. Questionário de Atividades de Autocuidado com o Diabetes (QAD) na amostra estudada $(n=42)$

\begin{tabular}{|c|c|c|c|c|}
\hline Itens & Variáveis & Média \pm & DP & EP \\
\hline 1.1 & $\begin{array}{l}\text { Seguiu uma alimentação sau- } \\
\text { dável }\end{array}$ & 5,29 & 2,52 & 0,39 \\
\hline 1.2 & Seguiu a orientação alimentar & 4,29 & 3,06 & 0,47 \\
\hline 2.1 & $\begin{array}{l}\text { Ingeriu cinco ou mais por- } \\
\text { ções de fruta e/ou vegetais }\end{array}$ & 4,79 & 2,61 & 0,40 \\
\hline 2.2 & $\begin{array}{c}\text { Evitou ingerir alimentos ricos } \\
\text { em gordura }\end{array}$ & 3,24 & 2,80 & 0,43 \\
\hline 2.3 & $\begin{array}{l}\text { Evitou ingerir alimentos do- } \\
\text { ces }\end{array}$ & 4,86 & 2,19 & 0,34 \\
\hline 3.1 & $\begin{array}{l}\text { Praticou atividade durante } \\
\text { pelo menos } 30 \text { minutos }\end{array}$ & 2,36 & 2,44 & 0,38 \\
\hline 3.2 & $\begin{array}{c}\text { Praticou exercício físico es- } \\
\text { pecífico }\end{array}$ & 1,38 & 2,13 & 0,33 \\
\hline 4.1 & Avaliou o açúcar no sangue & 2,69 & 3,11 & 0,48 \\
\hline 4.2 & $\begin{array}{c}\text { Avaliou o açúcar no sangue o } \\
\text { número de vezes recomen- } \\
\text { dado }\end{array}$ & 5,33 & 2,89 & 0,75 \\
\hline 5.1 & Examinou os pés & 6,02 & 2,28 & 0,35 \\
\hline
\end{tabular}




\begin{tabular}{ccccc}
5.2 & $\begin{array}{c}\text { Examinou dentro dos sapatos } \\
\text { antes de calçá-los }\end{array}$ & 4,82 & 3,12 & 0,48 \\
$5.3 \quad \begin{array}{c}\text { Secou os espaços entre os de- } \\
\text { dos do pé depois de lavá-los } \\
\text { Tomou os medicamentos da } \\
\text { diabetes conforme foi indi- } \\
\text { cado }\end{array}$ & 5,88 & 2,54 & 0,39 \\
$6.1 \quad \begin{array}{c}\text { Tomou injeções de insulina } \\
\text { conforme foi indicado }\end{array}$ & 6,40 & 1,68 & 0,48 & 0,07 \\
$6.2 \quad \begin{array}{c}\text { Tomou onumero indicado de } \\
\text { comprimidos da diabetes }\end{array}$ & 6,74 & 1,17 & 0,18 \\
\hline
\end{tabular}

Fonte - Elaborada pelos autores

O questionário de autocuidado apresentou que a maior adesão ao tratamento se deu pelo gerenciamento de medicamentos conforme a orientação prescrita pelos profissionais de saúde, seja de uso oral ou da insulina. Na sequência, o autocuidado em examinar e secar os espaços entre os dedos dos pés para evitar fissuras ou outras complicações, assim como avaliar o açúcar no sangue o número de vezes recomendado para insulínicos e seguir uma alimentação saudável, principalmente evitando doces, foram as outras atividades em que os participantes mais aderiram. Por sua vez, a menor adesão foi no domínio de atividade física, seguido do item "avaliar o açúcar no sangue" no geral.

Ao analisar os hábitos tabágicos, destaca-se um número expressivo de pessoas que nunca haviam fumado $(n=23)$ ou de ex-fumantes $(n=16)$. Apenas três pessoas informaram ter fumado na última semana, com variações entre 1 a 10 cigarros por dia.

O instrumento padronizado de avaliação utilizado permitiu retratar a adesão do indivíduo ao seu plano de cuidados, ao identificar o nível de engajamento das pessoas com diabetes em atividades de autocuidado, além de identificar indicadores que possam ser melhor gerenciados nos programas de educação continuada em saúde.

\section{DISCUSSÃO}

O engajamento em diferentes atividades de autocuidado é necessário para a adoção de um estilo de vida que seja adequado para o controle do diabetes mellitus. Este fato revela que o plano de cuidados deve ser pensado de forma colaborativa entre os profissionais que buscam a promoção da saúde e a prevenção de incapacidades. Além disso, o sucesso no tratamento está associado a considerar a pessoa diabética como um sujeito ativo e corresponsável pelo seu autocuidado ${ }^{4}$.

A amostra da pesquisa apontou para uma maior participação de mulheres com baixa escolaridade e sem hábitos tabágicos. A prevalência desta doença crônica é maior em mulheres ${ }^{6}$, e a literatura ainda informa que este gênero é o que mais procura os serviços de saúde ${ }^{7}$, o que pode explicar o quantitativo maior nesta pesquisa. Houve um equilíbrio no número de diabéticos em relação aos grupos etários, com uma discreta participação maior de pessoas idosas. Dados da escolaridade e da idade apresentada pelos participantes corroboram com os dados epidemiológicos sobre o diabetes mellitus ${ }^{1,8}$. Pesquisas ainda relatam uma correlação positiva entre o nível de escolaridade e a adesão ao regime terapêutico ${ }^{9}$, assim como aqueles que possuem o maior nível de escolaridade tem mais facilidade em aderir à prática de atividades físicas ${ }^{10}$.

De forma positiva, a maioria dos entrevistados não possuíam o hábito de fumar, o que atua como fator protetivo para a condição de saúde, uma vez que o tabagismo é fator de risco para o desenvolvimento de neuropatia diabética (com aumento de chance para amputações) e doenças cardiovasculares ${ }^{11}$.

$\mathrm{Na}$ análise dos dados, o resultado da pesquisa constatou maior adesão ao gerenciamento medicamentoso. Estes achados também foram encontrados nos estudos de Boas et al ${ }^{12}$ e Broadbentet $a l^{13}$. Nos cuidados em saúde, incorporar na rotina do diabético o tratamento com fármacos parece ser a atividade de autocuidado mais tradicional e simples de ser feita, uma vez que não são exigidas muitas alterações no estilo de vida ${ }^{14,15}$. No entanto, a literatura científica é bastante clara ao informar que o controle dessa doença crônica só será eficaz se houver mudança no comportamento, integrando outros hábitos saudáveis em seu cotidiano ${ }^{16}$.

À semelhança, os participantes dessa pesquisa também demonstraram boa adesão no que se refere aos cuidados com os pés. O manejo apropriado do pé diabético inclui manter seca a região 
entre os dedos, as unhas com cortes rentes e verificar se há presença de micoses e fissuras ${ }^{17}$. É uma estratégia educativa em saúde amplamente divulgada na atenção básica, pois está atrelada à prevenção de ulcerações e possíveis amputações parciais ou totais do pé ${ }^{17}$, sobretudo em diabéticos com perda de sensação protetora (pela neuropatia) ou doença arterial periférica ${ }^{18}$. Estas complicações estão associadas ao aumento da morbimortalidade e elevados custos financeiros para os sistemas de saúde $^{19}$. Ademais, a presença de deficiência nas estruturas dos membros inferiores pode levar a limitações para realizar as atividades do cotidiano que exigem permanecer em pé ou em mobilidade, além de ter potencial para restringir a participação social $^{20}$.

Os participantes da pesquisa que eram insulínicos também apresentaram adesão satisfatória para a "análise do açúcar no sangue de acordo com as recomendações dos profissionais de saúde". A insulina é uma estratégia terapêutica eficaz para a melhoria da qualidade de vida dos usuários que dependem desse medicamento. Avaliar a glicemia capilar, além de possibilitar de forma imediata o monitoramento do controle metabólico, dá subsídios para as tomadas de decisões nos cuidados em saúde, caso os valores mensurados estejam fora dos padrões desejados $^{21}$.

No que tange ao autocuidado relacionado à alimentação, muitos participantes informaram evitar ingerir doces e alimentos ricos em gorduras, uma vez que estes produtos elevam o índice glicêmico e aumentam o risco de desenvolver ou agravar complicações cardiovasculares ${ }^{22}$. Os resultados se assemelham aos estudos de Santos et al ${ }^{5} \mathrm{e}$ Eid $e t a l^{23}$. É interessante destacar que, apesar de muitos participantes terem declarado seguir uma alimentação saudável, essa adesão é menor quando se questiona se o usuário segue a orientação nutricional prescrita pelos profissionais de saúde. Tal discrepância entre os resultados pode ser decorrente da falta de informação ou de compreensão sobre o que seria a nutrição "ideal" para pessoas com diabetes. Dar orientações sobre evitar produtos industrializados e processados, alimentos com açúcares refinados ou muito gordurosos, sem considerar o contexto e o ambiente do diabético, dificilmente resultará em mudanças no comportamento destas pessoas ${ }^{24}$.

Evidências científicas apontam que não há um plano nutricional universal que funcione para toda a população de pessoas com diabetes ${ }^{25}$. A adoção de hábitos alimentares mais saudáveis é consequência de um conhecimento prévio das preferências pessoais e culturais que envolvem as escolhas dos alimentos, associado à compreensão do poder financeiro para aquisição de produtos mais saudáveis e da capacidade da família em atuar como um facilitador para mudanças que irão gerar um melhor controle glicêmico e reduzir o risco para as comorbidades $^{4,25}$.

De forma preocupante, o grupo de participantes da pesquisa revelou baixa adesão à prática de atividade física. O mesmo ocorreu no estudo de Felix $e t a l^{26}$, realizado com pacientes com diabetes (tipo 1 e 2) que apresentaram uma frequência máxima de duas vezes por semana para realizar exercícios físicos. É preciso retomar que o grupo de participantes possuía baixa escolaridade e isso, em partes, pode justificar a baixa adesão à prática de atividades físicas, uma vez que estudos apontam para associações positivas entre as duas variáveis ${ }^{10}$.

Sabe-se que o padrão alimentar e a prática regular de atividade física de intensidade moderada (150 minutos por semana) são os hábitos que mais contribuem para o controle metabólico do diabetes, para evitar doenças cardiovasculares e até mesmo reduzir o uso de insulina ${ }^{14}$ e a incidência de diabetes do tipo $2^{1}$. Além de promover melhorias na qualidade de vida e atuar na redução do peso e do colesterol, exercitar-se regularmente reduz a hemoglobina glicada e os níveis plasmáticos da glicose em jejum ${ }^{27}$.

Uma ressalva deve ser feita para este resultado na pesquisa. Algumas coletas $(n=25)$ foram feitas no contexto da pandemia do novo coronavírus (SARS-CoV-2). Como anteriormente informado e aqui retoma-se, o questionário utilizado (QAD) 
considera a frequência da adesão ao autocuidado em sua última semana. Assim, a baixa frequência para realizar as atividades físicas pode ser decorrente das medidas restritivas de distanciamento sugeridas pelo Ministério da $\mathrm{Saúde}^{28}$, especialmente se considerar que muitos dos participantes era composto por pessoas idosas, ou seja, se constituía em grupo de risco para o Covid-19. Nesse sentido, seria interessante pesquisas futuras - pós-pandemia para compreender a real influência do contexto social na prática regular de atividades físicas.

Cautela também deve ser feita na interpretação dos resultados sobre os dados gerais de "avaliar o açúcar no sangue". Todos os participantes (insulínicos ou não) responderam a essa pergunta no questionário e a baixa adesão a essa prática de autocuidado pode ser devido à falta de recomendação médica para a realização de tal procedimento de forma frequente e sistemática para aqueles que não são insulínicos, o que gerou uma média menor que 3 dias na semana. Tais dados corroboram com o estudo de Neto et $a l^{29}$. Como mencionado anteriormente, quando foram analisados somente os insulínicos que receberam orientações para avaliação, a taxa de adesão foi satisfatória.

Há também um número expressivo de usuários $(n=27)$ que não possuem o aparelho em casa, pois o glicosímetro e as fitas para aferir a glicemia só são dispensadas pelo Sistema Único de Saúde àqueles que são insulínicos (que não corresponde a maior parte desta amostra, $n=15)$. Acresce aos dados, o fato de que muitos participantes (insulínicos ou não) realizavam essa atividade na Clínica da Família e, assim, pode ter ocorrido a redução de tal prática pela suspensão temporária do acompanhamento semanal dessas pessoas enquanto durar a pandemia do coronavírus. No entanto, é inegável a importância do uso de tecnologias para monitoramento da glicemia, pois elas informam resultados imediatos sobre as funções do corpo e contribuem para a tomada de decisões, em prol de um melhor cuidado em saúde ${ }^{30}$.
As limitações deste estudo decorrem no tamanho amostral e por ter sido restrita ao grupo de usuários que participava de um programa de educação e promoção em saúde para diabetes e hipertensão que acontecia em uma Clínica da Família. Assim, os dados não podem ser generalizados. Além disso, a estratégia metodológica adotada (estudo transversal) não permite associações de causa e efeito, sendo importante conduzir pesquisas longitudinais para compreender melhor a adesão das pessoas com diabetes às atividades de autocuidado, ao longo do tempo. No entanto, os resultados apresentados nesse estudo ressaltam a importância de uma abordagem multidimensional para a construção de um plano de cuidados, assim como fornecem indicativos das dimensões de autocuidado que ainda precisam ser trabalhadas para se obter resultados efetivos e resolutivos para o controle do diabetes.

\section{CONSIDERAÇÕES FINAIS}

Os usuários que participaram deste estudo apresentaram um bom nível de autocuidado, sobretudo no que se refere ao gerenciamento medicamentoso e cuidado com os pés. Entretanto, o domínio que corresponde à atividade física e o item de avaliar o açúcar no sangue precisam ser melhor investigados, considerando o contexto social, temporal e pessoal, para assim definir as estratégias mais adequadas para o plano de cuidados da pessoa com diabetes. Os resultados obtidos neste estudo servem de subsídios para a organização, planejamento e execução de ações interprofissionais voltadas à promoção e educação em saúde dos diabéticos.

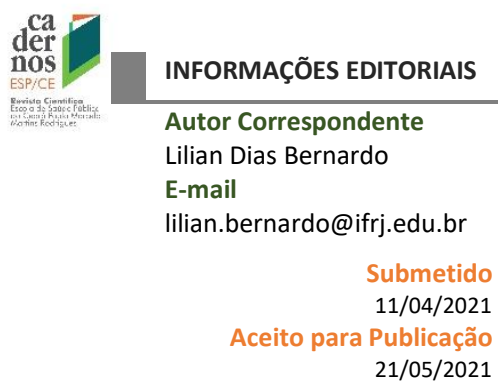




\section{REFERÊNCIAS}

1. Sociedade Brasileira de Diabetes. Diretrizes da Sociedade Brasileira de Diabetes 2019-2020. São Paulo (SP): Clannad; 2020.

2. Lu Y, Xu J, Zhao W, et al. Measuring self-care in personswithtype 2 diabetes: A systematic Rev.Eval Health Prof[Internet]

2016; 39(2):131-84. Disponível em: https://pubmed.ncbi.nlm.nih.gov/26130465/.

3. Oliveira CF, Arruda GMMS, Melo ALA, Barcelos AC. Cuidado interprofissional aos portadores de Hipertensão e Diabetes em um grupo de intervenção educacional e terapêutica. CadEspCeará. 2016; 10(2): 33-45. Disponível em:https://cadernos.esp.ce.gov.br/index.php/cadernos/article/view/105/112.

4. Ferreira CM, Soares EP, Carvalho GB, Silva AC. Intervenção educacional como ferramenta de gestão aos diabéticos.CadEsp Ceará. 2020; 14(1): 111 -114. Disponível em: https://cadernos.esp.ce.gov.br/index.php/cadernos/article/view/290/205.

5. Santos SD, Rocha MR, Moura Ionara H, et al. Atividade de autocuidado em pessoas com diabetes mellitus tipo 2. RevEnfermUFPE online [Internet]. 2019; 13:1-8. Disponível em:https://pesquisa.bvsalud.org/portal/resource/pt/biblio-1050782.

6. Silva DS, Laterza MC, Moreira OC, et al. Prevalência de diabetes mellitus em indivíduos atendidos pela estratégia saúde da família no município de Ubá-MG. RevBrasAtivFis e Saúde [Internet]. 2012; 17(3):195-199. Disponível em:

https://rbafs.org.br/RBAFS/article/view/ 1858/1698

7. Gomes R, Nascimento EF, Araújo FC. Por que os homens buscam menos os serviços de saúde do que as mulheres? As explicações de homens com baixa escolaridade e homens com ensino superior. CadSaúde Pública [Internet]. 2007; 23(3):565-574. Disponível em:http://www.scielo.br/scielo.php?script=sci_arttext\&pid=S0102-311X2007000300015\&lng=en.

8. Flor LS, Campos MR. Prevalência de diabetes mellitus e fatores associados na população adulta brasileira: evidências de um inquérito de base populacional. RevBrasEpid[Internet]. 2017; 20(1):16-29. Disponível em:http://www.scielo.br/sci-

elo.php?script=sci_arttext\&pid=S1415-790X2017000100016\&lng=en.

9. Carvalho AZFHT, Andrade JU, Lima TM, et al. Adesão ao regime terapêutico de pacientes com diabetes mellitus: análise de atividades de autocuidado. Braz. J. ofDevelop[Internet]. 2020; 6(7): 48115-48129. Disponível em: file:///C:/Users/Lilian\%20Dias/Desktop/13405-34775-1-PB.pdf.

10. Streb AR, Leonel LS, Silva CS, et al. Associação entre a prática de atividade física em diferentes domínios e o uso de insulina em adultos e idosos com diabetes no Brasil. Ciênc Saúde Col [Internet]. 2020; 25(11): 4615-4622. Disponível em: https://www.scielosp.org/pdf/csc/2020.v25n11/4615-4622/pt.

11. Boell JEW, Silva DMGV, Guanilo MEE, et al. Resiliência e autocuidado em pessoas com diabetes mellitus. Texto Contexto Enferm[Internet]. 2020; 29:e20180105. Disponível em: http://www.scielo.br/scielo.php?script=sci_arttext\&pid=S0104-

07072020000100327\&lng=en

12. Villas-Boas LCG, Foss MC, Freitas MCF, et al. Relação entre suporte social, adesão ao tratamento e controle metabólico de pacientes com diabetes mellitus. RevLatino-Am. Enfermagem [Internet]. 2012; 20(1):52-58. Disponível em:http://www.scielo.br/scielo.php?script=sci_arttext\&pid=S0104-11692012000100008\&lng=en.

13. Broadbent E, Donkin L, Stroh JC. Illnessandtreatmentperceptions are associatedwithadherencetomedications, diet, andexercise in diabeticpatients. Diabetes Care [Internet]. 2011; 34(2):338-40. Disponível em:https://pubmed.ncbi.nlm.nih.gov/21270191/. 14. Trevizani FA, Doreto DT, Lima GS, et al. Atividades de autocuidado, variáveis sociodemográficas, tratamento e sintomas depressivos em idosos com diabetes mellitus. Rev. Bras. Enferm. [Internet]. 2019; 72 (2):22-29. Disponível em:http://www.scielo.br/scielo.php?script=sci_arttext\&pid=S0034-71672019000800022\&lng=en.

15. Faria HTG, Rodrigues FFL, Zanetti ML, et al. Fatores associados à adesão ao tratamento de pacientes com diabetes mellitus. Acta Paul Enferm[Internet]. 2013; 26(3):231-237. Disponível em:http://www.scielo.br/scielo.php?script=sci_arttext\&pid=S010321002013000300005\&lng=en.

16. Gabbay RA, Durdock K. Strategiestoincreaseadherencethrough diabetes technology. J Diabetes SciTechnol [Internet]. 2010; 4(3):661-5. Disponível em:https://pubmed.ncbi.nlm.nih.gov/20513334/.

17. Perdomo CR, Romero AP, Vélez MR. Conhecimentos e práticas para a prevenção do pé diabético. Rev Gaúcha Enferm [Internet]. 2019; 40:e20180161. Disponível em:http://www.scielo.br/scielo.php?script=sci_arttext\&pid=S1983-

$14472019000100408 \& \operatorname{lng}=$ en.

18. Schaper NC, van Netten JJ, Alpelqvist J, et al. Practicalguidelinesonthepreventionand management ofdiabeticfootdisease. Diabetes Metab Res Rev [Internet]. 2020; 36(1);e3266. Disponível em:https://pesquisa.bvsalud.org/controlecancer/resou$\mathrm{rce} / \mathrm{pt} / \mathrm{mdl}-32176447$.

19. Eleftheriadou I, Samakidou G, Tentolouris A, et al. Nonpharmacological management ofdiabeticfootulcers: anupdate. Int. J. LowExtremWounds [Internet] 2020; 19:15347346209663561. Disponível em:https://pubmed.ncbi.nlm.nih.gov/33073653/.

20. Foong HF, Kyaw BM, Upton Z, et al. Facilitatorsandbarriersofusing digital technology for the management ofdiabeticfootulcers: A qualitativesystematicreview. IntWound J [Internet]. 2020; 17:1266-1281. Disponível em: https://pubmed.ncbi.nlm.nih.gov/32390305/.

21. Maia FFR, Araújo LR. Impacto do sistema de monitorização contínua da glicose em pacientes diabéticos. RevAssocMedBras[Internet]. 2006; 52(6): 395-400. Disponível em: https://www.scielo.br/pdf/ramb/v52n6/a17v52n6.pdf.

22. Lira MCA, de Souza NMM, Burgos MGPA. Prevalência de fatores de risco para doenças cardiovasculares em diabéticas. NutrClín Diet Hosp [Internet]. 2017; 37(1):75-81. Disponível em:https://revista.nutricion.org/PDF/mayaramenezes.pdf. 
23. Eid LP, Leopoldino SAD, Oller GASAO, et al. Fatores relacionados às atividades de autocuidado de pacientes com diabetes mellitus tipo 2. EscAnna Nery [Internet]. 2018; 22(4):e20180046. Disponível em: http://www.scielo.br/scielo.php?script=sci_arttext\&pid=S1414-81452018000400201\&lng=en.

24. Pereira J, Frizon E. Adesão ao tratamento nutricional de portadores de diabetes mellitus tipo 2: uma revisão bibliográfica. RevAssocBras Nutri [Internet]. 2018; 8(2):58-66. Disponível em:https://www.rasbran.com.br/rasbran/article/view/330.

25. Evert AB, Boucher JL, Cypress M, et al. Nutritiontherapyrecommendations for the management ofadultswith diabetes. Diabetes Care [Internet]. 2013; 36(11):3821-42. Disponível em:https://pubmed.ncbi.nlm.nih.gov/24107659/.

26. Felix LG, Lemos LGH, Negreiros RV, et al. Adesão às práticas de autocuidado entre pessoas idosas com diabetes mellitus. Anais IV CIEH [Internet]. 2015; 2(1): 1-7. Disponível em:http://editorarealize.com.br/artigo/visualizar/12017.

27. Loureiro LAR, Afonentão MIV, Ribeiro OS, et al. Exercício físico em pessoa com diabetes: revisão sistemática de literatura. RevPortEnferReabi [Internet]. 2019 jun; 2(1):19-26. Disponível em:https://www.researchgate.net/publication/334095829_Exercicio_fisico_em_pessoas_com_diabetes_revisao_sistematica_de_literatura.

28. Ministério da Saúde (BR). Portaria n 454 , de 20 de março de 2020. Declara, em todo o território nacional, o estado de transmissão comunitária do coronavírus (covid-19). Diário Oficial da União. 2020 Mar. 20.

29. Neto JDM, Santos-de-Araújo AD, Santana TCFS, et al. Adesão dos pacientes de diabetes mellitus às atividades de autocuidado. RevInvestBiom[Internet]. 2018; 10(2):132-14. Disponível em: https://www.researchgate.net/publication/332459001_Adesao_dos_pacientes_de_diabetes_mellitus_as_atividades_de_autocuidado.

30. Bernardes MS, Santana C da S. Health monitoringanddecision-makingbyolderadults. Gerontechnology [Internet]. 2018; 17(4):238-244. Disponível em:http://dx.doi.org/10.4017/gt.2018.17.4.005.00. 